\title{
Immobilization and Encapsulation of Micro- and Nano- Objects with Electron Transparent Graphene Oxide membranes
}

\author{
Alexander Yulaev ${ }^{1,3}$, Alexey Lipatov ${ }^{2}$, Alexander Sinitskii ${ }^{2}$, and Andrei Kolmakov ${ }^{3}$ \\ ${ }^{1}$ Department of Materials Science and Engineering, University of Maryland, College Park, MD 20742, \\ USA \\ ${ }^{2}$ Department of Chemistry, University of Nebraska - Lincoln, Lincoln, NE, 68588, USA \\ ${ }^{3}$ Center for Nanoscale Science and Technology at NIST, 100 Bureau Drive, Gaithersburg, MD \\ 20899-6204 USA.
}

The encapsulation of objects with protective layers is employed in anticorrosive coatings of the metals [1], conformal coatings in microelectronics [2], micro encapsulation of chemicals for drug delivery [3] and etc. In many research and histological practices the microscopy access to the coated sample is desirable. We report here on facile methodology for immobilization and encapsulation of objects at micro- and nano- scales by means of nanometer thin and electronically transparent graphene oxide (GO) membranes. There are several features that make GO membranes advantageous for microscopy friendly encapsulation: (i) Low Z number for GO and, therefore, its high electron transparency, (ii) facile formation of the GO membranes at liquid-gas-solid interfaces coupled with simple and cost-effective wet protocols; (iii) its mechanical stiffness upon drying [5].

The mechanism of GO film formation over the core object is depicted in fig. 1. GO water solution was drop casted onto specimen. Due to its amphiphilic properties, GO flakes segregate at the interfaces forming water permeable membranes (fig. 1,a). Water almost freely penetrates through overlapping GO flakes to the ambient [4] and droplet gradually evaporates (fig. 1, b) leaving condensing membrane covering the sample of interest. The evaporation of water along with membrane contraction results in pressure build up inside the encapsulated volume (fig. 1,c) and finally its complete settlement over the core object (fig. 1, d). To demonstrate the versatility of this techniques to fix and encapsulate microscopic object we tested a number of samples i.e.: $\mathrm{SnO}_{2}$ nanofiber (fig. 1, e-f), $\mathrm{Hg}$ liquid droplet (fig. 2, a), hydrogen gas bubbles (fig. 2, b), and biological nano- an micro- objects. Finally, encapsulation of micro-objects on TEM membrane has been performed (fig. 2, c).

The GO coverage of the solid samples as $\mathrm{SnO}_{2}$ nanofibers on aluminum substrate has characteristic "wrapping" behavior, exhibiting free suspended regions of stretched GO at the peripheral contour of the sample. The encapsulation of the incompressible liquid object like mercury droplet resulted in significant distortion of the shape of the droplet due to tension forces developing in the GO membrane upon drying. We used hydrogen bubbles encased by GO film to explore membrane induced resolution deterioration during SEM (Figure 2b). It clearly demonstrated the contrast deterioration due to electron scattering by GO membrane. Finally, Figure 2c demonstrates the usefulness of GO membranes to gentle immobilization /encapsulation of micro-objects on the TEM grids for HRTEM studies. We envision that this technique can be successfully applied in histological practices, forensic studies, bio-medical research, as well as in other arrears where isolation of toxic or radioactive artifacts is required [5].

\section{References:}

[1] P. A. Sorensen et al, Journal of Coatings Technology and Research 6 (2) (2009), pp. 135-176. 
[2] Armin G. Aberle, Progress in Photovoltaics: Research and Applications 8 (5) (2000), pp. 473-487.

[3] Rainer H. Muller et al, European Journal of Pharmaceutics and Biopharmaceutics 50 (1) (2000), pp. 161-177.

[4] R. Nair et al, Science 335 (6067) (2012), pp. 442-444.

[5] D.A. Dikin et al, Nature 448 (7152) (2007), pp. 457-460.

[6] The authors are thankful to Profs J. Huang (Northwestern University), Dr. Nina Kovtyukhova (Penn State University) for fruitful discussions and GO samples. We appreciate the help of Prof. Vjollca Konjufca and Prof. Marjorie Brooks (both SIUC) in handling biological samples.
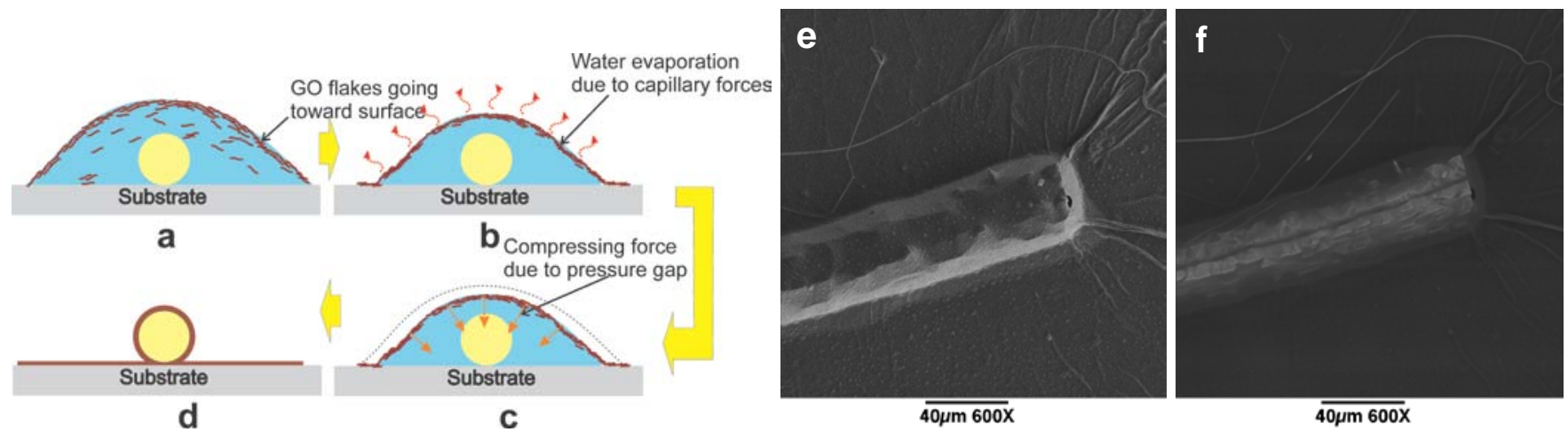

Figure 1. a) GO flakes segregate at the interfaces due to their amphiphilicity; b) water penetrates through GO membrane, and droplet gradually evaporates; c) pressure differential builds up between interior and ambient as a result of water evaporation; and d) the final stage of GO encapsulation: object is completely encased by solidified GO layer; e) SEM micrographs of GO covering NW tip, $\mathrm{E}_{\mathrm{b}}=5 \mathrm{keV}$; f) the same specimen at $E_{b}=20 \mathrm{keV}$.

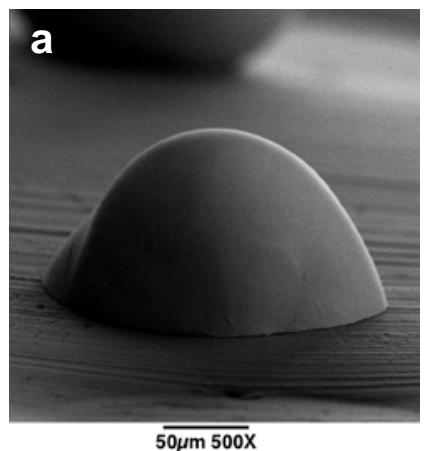

$\overline{50 \mu \mathrm{m} \mathrm{500x}}$

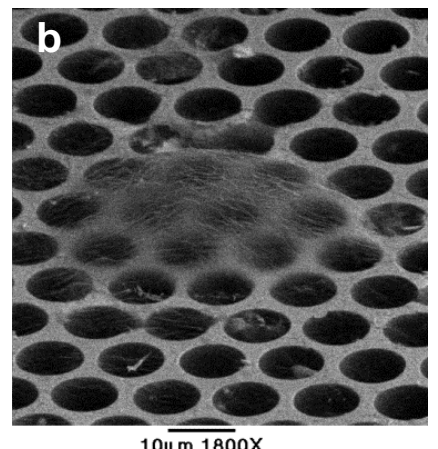

$10 \overline{\mathrm{m} 1800 \mathrm{x}}$

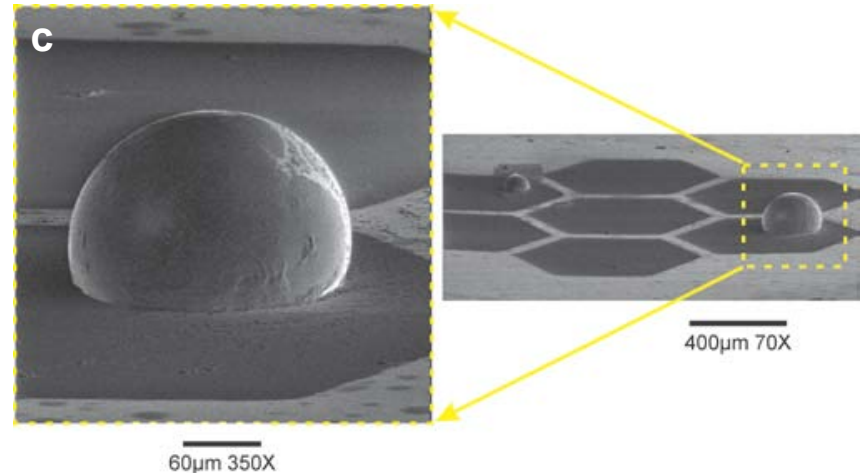

$\overline{604 m 350 x}$

Figure 2. a) SEM of GO encapsulated mercury micro-droplet on aluminum substrate at $E_{b}=15 \mathrm{keV}$; b) SEM image of GO bubble specimen taken at $E_{b}=10 \mathrm{keV}$; c) Tilted SEM images at $\mathrm{E}_{\mathrm{b}}=1 \mathrm{keV}$ of $\mathrm{Hg}$ droplets adhered to TEM membrane by means of GO drop casted membrane, inset: encapsulated immobilized mercury droplets on TEM grid. 